\title{
Double Star Speckle Interferometry with the 3.5-m Telescope at Calar Alto (Almería, Spain)
}

\author{
J.A. Docobo ${ }^{1}$, V.S. Tamazian ${ }^{1}$, M. Andrade ${ }^{1}$, J.F. Ling ${ }^{1}$, \\ Y.Y. Balega ${ }^{2}$, J.F. Lahulla ${ }^{3}$, A. Maximov ${ }^{2}$ \\ and J.R. González-Romay ${ }^{1}$ \\ ${ }^{1}$ Astronomical Observatory R. M. Aller, Universidade de Santiago de Compostela, 15782 \\ Santiago de Compostela, Spain \\ email: oadoco@usc.es \\ ${ }^{2}$ Special Astrophysical Observatory, Nizhnij Arkhyz, Karachai-Cherkesia 369167, Russia \\ ${ }^{3}$ Observatorio Astronómico Nacional, Calle Alfonso XII 3, 28014 Madrid, Spain
}

\begin{abstract}
The first results of speckle interferometry carried out with the 3.5-m telescope of the C.A.H.A. (Almería, Spain) during a run in July, 2005 are presented. Forty nine stars with separations between 0 .'058 and 2.' 1 were observed under good seeing conditions. On the basis of these observations three improved orbits are presented. The Time Allocation Committee's report with a high scientific qualification to our proposal is included. It confirms the relevance of binary and multiple star research in modern astronomy and the great significance of large telescopes in this kind of studies as well.
\end{abstract}

Keywords. instrumentation: interferometers, methods: data analysis, techniques: interferometric, astrometry, (stars:) binaries: visual, stars: fundamental parameters

\section{Introduction}

The Astronomical Observatory R.M. Aller (OARMA) of the University of Santiago de Compostela (Galicia, Spain) constructed its speckle camera through a public tender of the Autonomous Government (Xunta de Galicia) in 1998. This instrument was developed in cooperation with the Special Astrophysical Observatory (SAO) of Russia and attached to the 1.52-m telescope at Calar Alto (Almería, Spain) during the first observational runs (Docobo et al. 2001, 2004, 2006).

In a second stage, we requested observational time at larger aperture telescopes (concretely, the 3.5-m telescope at Calar Alto and the 6.0-m telescope at SAO) with the aim to take more advantage of the camera.

The 3.5-m telescope Time Allocation Committee's evaluation of our proposal (shown in Figure 1) confirms the high interest of double and multiple stars research in modern Astronomy.

\section{The camera}

The main module (see Figure 2) contains a pair of interchangeable microscope objectives with magnifications $8 \times$ and $20 \times$, which are used to sample the size of individual speckles (about $4 \AA$ at $500 \mathrm{~nm}$ at the $\mathrm{f} / 8$ Cassegrain focus of the $1.52-\mathrm{m}$ telescope) to a detector's pixel, with a size of $13.4 \mu \mathrm{m}$. The corresponding scale on the detector is 0 "' 028 or $0 . \prime 011$ per pixel with total fields of view of 5.6 and 14.3 arcsec square. We normally use a $20 \times$ microscope objective. 


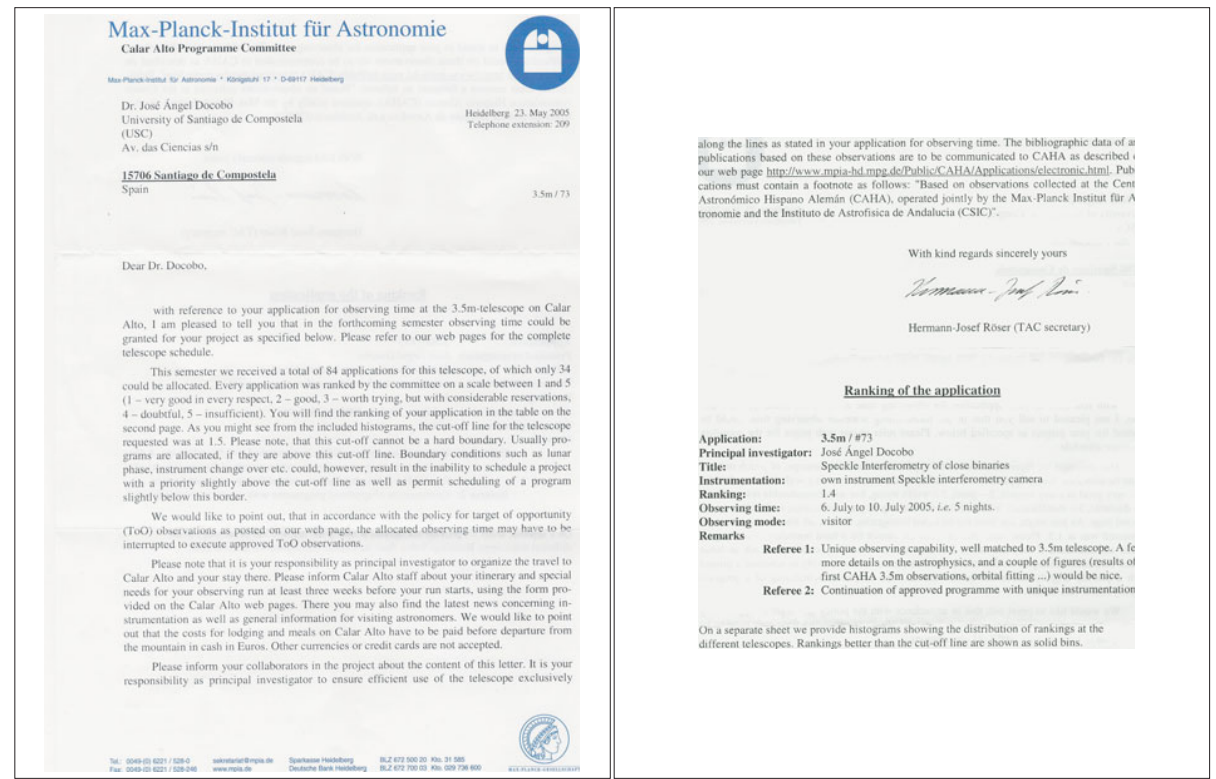

Figure 1. 3.5-m time Allocation Commitee response (CAHA).

In order to provide the exposures in the range 5 to $40 \mathrm{~ms}$, an Uniblitz remote-controlled electronic shutter placed in front of the microscope objective is synchronized with the CCD detector readout.

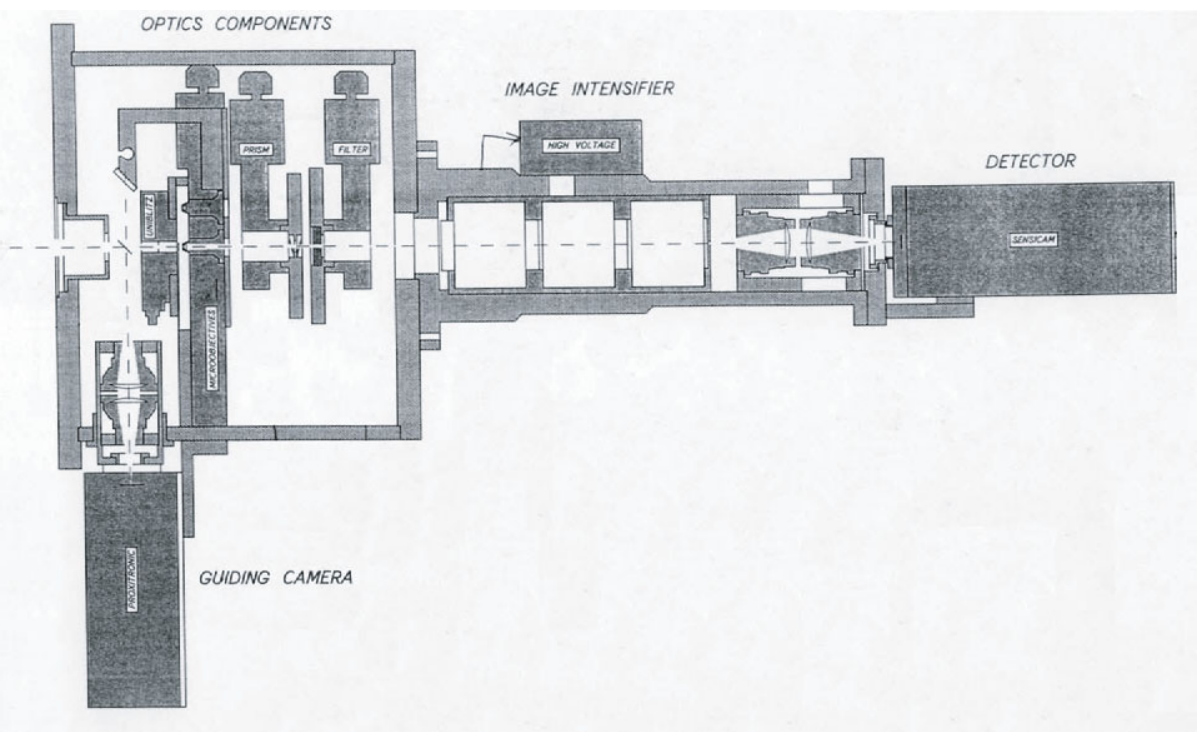

Figure 2. Speckle camera scheme.

Data are routinely obtained through the 520/24-nm filter but a filter wheel assembly also includes 600/50-nm and 660/40-nm filters. In addition, a set of 4 zero-mean-deviation prisms, mounted on a rotation stage, is used for atmospheric dispersion compensation at different zenithal angles (for the $20^{\circ}$ radius from the zenith, the clear aperture is selected). 
The detector consists of a PCO Computer Optics (Germany) Sensicam CCD camera with $1280(\mathrm{H}) \times 1024(\mathrm{~V})$ pixels of $6.7 \times 6.7 \mu \mathrm{m}$, optically coupled by means of a pair of $\mathrm{f} / 1.5$ transfer lenses to a 3-stage electrostatically-focused image intensifier. The input 24-mm photocathode of the intensifier has an S-25 spectral response with a peak sensitivity of $12 \%$ at $510 \mathrm{~nm}$, and about $2 \%$ sensitivity is still available at $800 \mathrm{~nm}$. For faster readout we use the sampling of speckle images to $512 \times 512$ pixels. The dynamic range of the system is limited by the 12-bit digitalization. Single photoelectron events are recorded by the system with a signal-to-noise ratio of about 30 .

\section{Results}

\subsection{Measurements}

By means of speckle interferometry, the Rayleigh's resolution limit of 31 mas for a 3.5-m telescope (see Figure 3) can be achieved, allowing to observe close visual pairs.

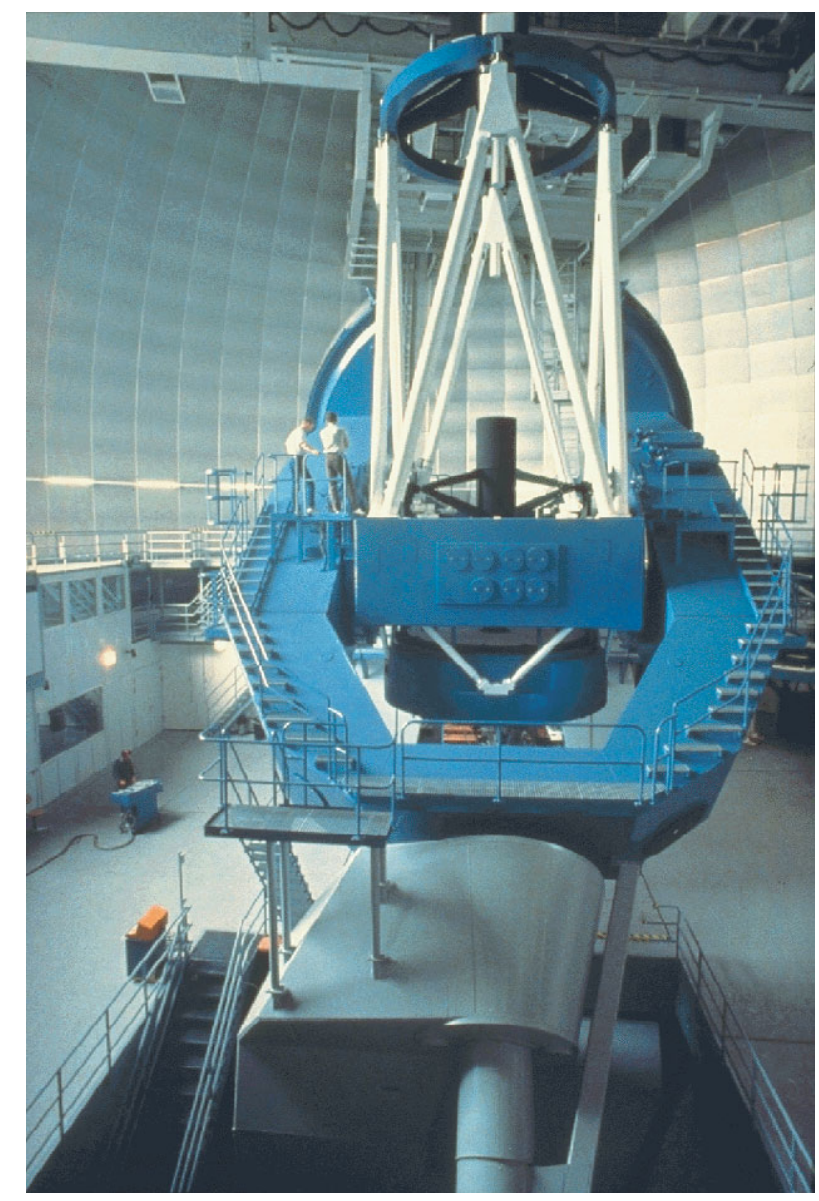

Figure 3. 3.5-m telescope at Calar Alto.

In Figure 4, the angular separation distribution of the measurements performed during the last campaign is shown. The $27 \%$ of them have angular separations less than $00^{\prime \prime} 1$ and only $18 \%$ have more than 0.5 . The mean error in angular separation is about $0 . \prime 003$, whereas those for position angle is 0.5 . 


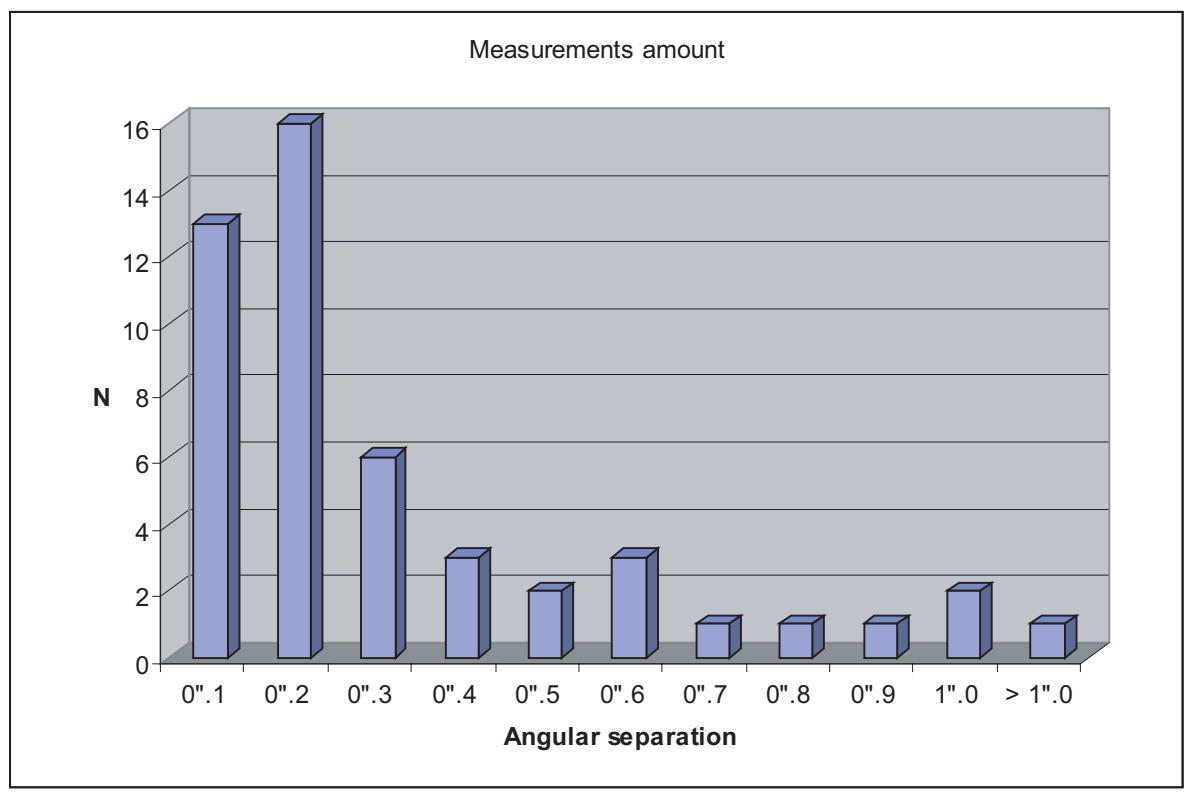

Figure 4. Angular separation distribution.

\subsection{Orbits}

A significant amount of the obtained measurements confirm a systematic departure of observational residuals from predicted positions for some orbits. We have recalculated three of them (A 225, A 2189, and COU 1962, their orbits are shown in Figures 5, 6, and 7 , respectively) using the analytical method of Docobo (1985) and taking into account both micrometric and speckle observations indicated by filled circles and asterisks, respectively. Our 3.5-m measurements are indicated by red asterisks, all being close to the minimal apparent separation. The orbital elements of these orbits have been submitted for publication in the IAU Commission 26 Information Circular 159.

\subsubsection{A 225 (WDS 16198+2647)}

The previous orbit was calculated by Heintz (1982). Our measurement gives residuals of -5.3 and 0 ' 030 in position angle and angular separation, respectively. Using our near periastron measurement, we have calculated a new, less eccentric orbit (e=0.49 vs 0.64).

\subsubsection{A 2189 (WDS 17563+0259)}

In this case, we have slightly improved a previous orbit (Docobo\&Costa 1991).

\subsubsection{COU 1962 (WDS 20311+3333)}

For this star, the previous orbit (Docobo\&Ling 1999) has been improved by taking into account a unique first quadrant measurement. Its period has grown to 9.48 years.

\section{Remarks}

In view of our successful results, we intend to use this telescope for the follow-up observations of close binary and multiple systems of special astrophysical interest. 


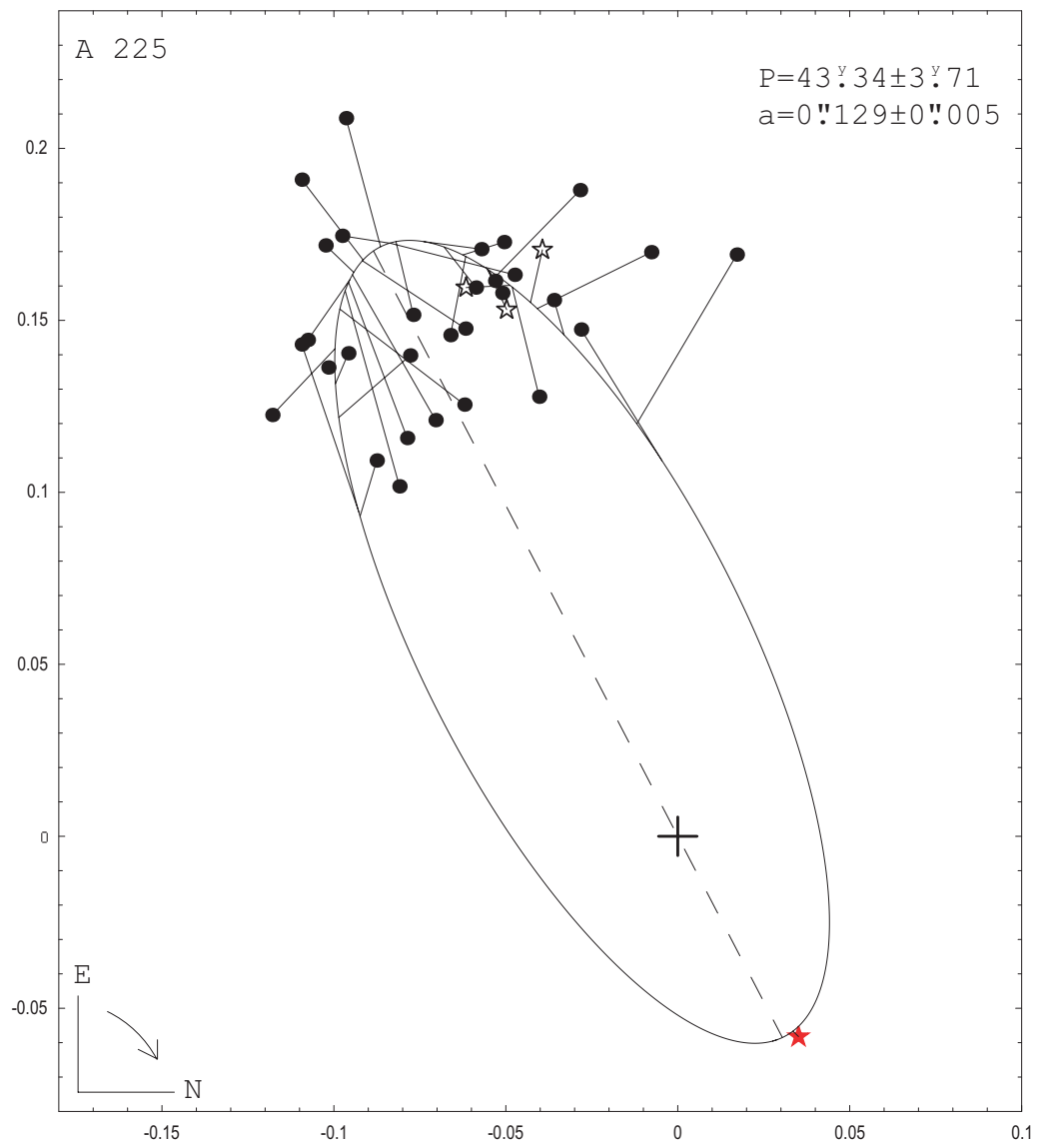

Figure 5. Apparent orbit of A 225.

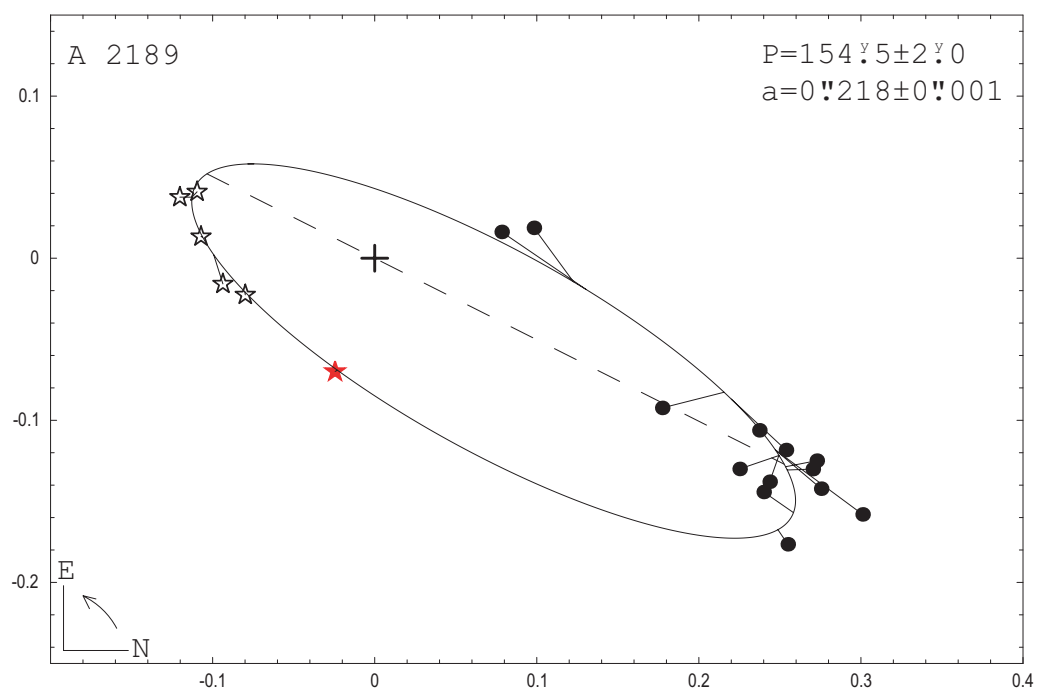

Figure 6. Apparent orbit of A 2189. 


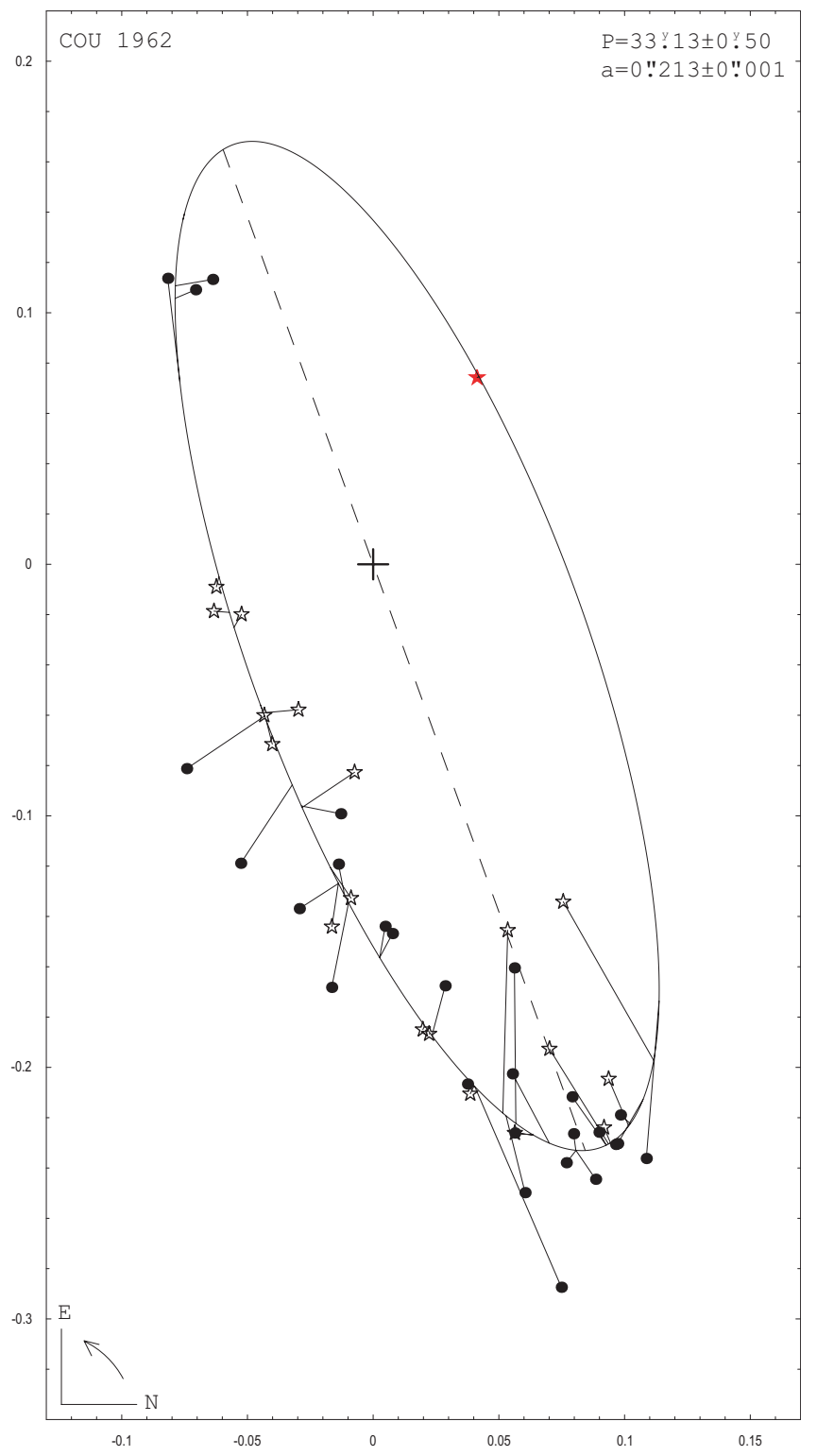

Figure 7. Apparent orbit of COU 1962.

\section{References}

Docobo, J.A. 1985, Celestial Mechanics, 36, 143

Docobo, J.A., Andrade, M., Tamazian, V.S., Costado, M., \& Lahulla, J.F. 2006 [Submitted to Rev. Mexicana AyA]

Docobo, J.A., Andrade, M., Ling, J.F., Prieto, C., Tamazian, V.S., Balega, Y.Y., Blanco, J., Maximov, A.F., Lahulla, J. F., \& Álvarez, C. 2004, AJ, 127, 1181

Docobo, J. A., Tamazian, V.S., Balega, Y. Y., Blanco, J., Maximov, A.F., \& Vasyuk, V. A. 2001, A\&A, 366, 868

Docobo, J.A. \& Costa, J.M. 1991, Inf. Circ. 113

Docobo, J.A. \& Ling, J.F. 1999, ApJS, 120, 41

Heintz, W.D. 1982, A\&AS, 47, 569 